\title{
Key Role of Some Specific Occupied Molecular Orbitals of Short Chain n-Alkanes in Their Surface Tension and Reaction Rate Constants with Hydroxyl Radicals: DFT Study
}

\author{
Mikhail Yu Gorbachev, Natalia N. Gorinchoy*, Ion Arsene \\ Department of Physical and Quantum Chemistry, Institute of Chemistry, Academy of Sciences of Moldova, Kishinev, \\ Republic of Moldova \\ Email: *ngorinchoy@yahoo.com
}

How to cite this paper: Gorbachev, M.Y., Gorinchoy, N.N. and Arsene, I. (2021) Key Role of Some Specific Occupied Molecular Orbitals of Short Chain n-Alkanes in Their Surface Tension and Reaction Rate Constants with Hydroxyl Radicals: DFT Study. International Journal of Organic Chemistry, 11, 1-13.

https://doi.org/10.4236/ijoc.2021.111001

Received: December 11, 2020

Accepted: January 26, 2021

Published: January 29, 2021

Copyright $\odot 2021$ by author(s) and Scientific Research Publishing Inc. This work is licensed under the Creative Commons Attribution International License (CC BY 4.0).

http://creativecommons.org/licenses/by/4.0/

\begin{abstract}
Basing on the DFT calculations we propose the new theoretical model which describes both the surface tension $\sigma$ of the short chain n-alkanes at their normal boiling points and their reaction rate constants with hydroxyl radicals $\mathrm{OH}^{\bullet}$ (at $297 \pm 2 \mathrm{~K}$ ) on the basis of their molecular orbital electronic characteristics. It has been shown that intermolecular dispersion attraction within the surface liquid monolayer of these compounds, as well as their reaction rate constants $k$ with $\mathrm{OH}^{\bullet}$ radicals are determined by the energies $E_{\text {orb }}$ of the specific occupied molecular orbitals which are the same in the determination of both the above physico-chemical characteristics of the studied n-alkanes. The received regression equations confirm the theoretically found dependences between the quantities of $\sigma$ and $k$ and the module $\left|E_{o r b}\right|$. For the compounds under study this fact indicates the key role of their electronic structure particularities in determination of both the physical (surface tension) and the chemical (reaction rate constants) properties.
\end{abstract}

\section{Keywords}

Surface Tension, Reaction Rate Constants, n-Alkanes, Hydroxyl Radicals, Dispersion Attraction, Orbital Controlled Interactions, DFT Calculations

\section{Introduction}

As is known, many physicochemical properties of chemical compounds to a large extent related to some characteristic features of their electronic structure, including, first of all, certain occupied frontier Molecular Orbitals (MOs). On 
the one hand, these MOs can determine such physical properties of liquid chemical compounds as surface tension [1] [2], viscosity and electrical conductivity [3], aqueous solubility [4], etc. On the other hand, they are responsible for the wide range of chemical properties: the reaction rate constants [5], enthalpies of formation [6], relative stability of isomers [7], pKa values [8], etc. Here it is important to note, that the above-mentioned occupied MOs are not necessarily the highest occupied MOs (HOMOs) of the compounds under study. So, in the works [1] [2] [8] it was shown that the occupied MOs which are responsible for the studied physicochemical properties can be HOMO-1, HOMO-2, etc.

In particular, in our previous work [1] it has been shown that the surface tension $\sigma$ of the near-boiling liquids whose molecules possess $\pi \sigma^{\star}$-hyperconjugated electronic structures is determined by certain occupied MOs which explicitly describe this $\pi \sigma^{\star}$-hyperconjugation of the methyl groups of each molecule with its $\pi$-electron system. As in the present work we study the surface tension of boiling n-alkanes, one can assume that this quantity may depend on some occupied MO which contains $1 \mathrm{~s}$ atomic orbitals (AOs) of all the hydrogen atoms of the studied hydrocarbons. This requirement to the above MO follows from the fact that interaction of neighboring molecules in liquid n-alkanes is being realized via $\mathrm{H}-\mathrm{H}$ interactions because of carbon atoms' spatial shielding.

The frontier molecular orbitals can also play an important role in the determination of the reaction rate constants for the interaction of structurally related compounds with a common reagent. So, for instance, it in the work [5] the energies of these MOs of various organic compounds fit very well for the quantitative description of the reaction rate constants for their interaction with the free radicals $\mathrm{O}_{2}^{*}$ and $\mathrm{HO}_{2}^{\circ}$. At the same time there is a great number of the experimental data, describing the reaction rate constants of $\mathrm{n}$-alkanes with free hydroxyl radicals $\mathrm{HO}^{\bullet}$. It can be assumed that in the case of the interaction "hydrocarbon-OH radical" there is also a certain occupied molecular orbital, which is responsible for this process.

In this connection the main goals of the present work are twofold:

1) to understand the mechanism of formation of monomolecular surface layers for the liquids under consideration and to find an equation connecting the surface tension of $n$-alkanes at their normal boiling points with calculated molecular electronic structure parameters;

2) to clarify the transition state for the interaction "hydrocarbon-OH radical" and to determine the specific molecular orbitals of n-alkanes which play the crucial role in this reaction. Then, based on this knowledge, to establish a quantitative relationship between properties of these specific MOs and the reaction rate constants for the above interaction.

\section{Theory}

\subsection{Construction of Surface Layers and Surface Tension of Near-Boiling n-Alkanes}

In our previous work [1] it has been shown that the surface tension $\sigma$ of the 
near-boiling liquids whose molecules possess $\pi \sigma^{\star}$-hyperconjugated electronic structures is determined by the formation of some supramolecular $\pi \sigma^{\star}$-hyperconjugated electronic film-like system including all the molecules entering their surface monomolecular layers. For such liquids the values of $\sigma$ can be received from the following equation:

$$
\sigma=\chi\left|E_{o r b}\right| N V^{-2 / 3}\left(1-T / T_{c}\right),
$$

where $V$ is the molar volume of the liquid taken at a certain absolute temperature $T$, close to or equal to its normal boiling point, $T_{c}$ is its critical temperature, and $N$ is the total number of molecular centers which form one-particle $\pi \sigma^{\star}$ hyperconjugated electronic system. The quantity $\left|E_{o r b}\right|$ in Equation (1) is the absolute value of the energy of the molecular orbital (MO) responsible for $\pi \sigma^{*}$ hyperconjugation. The constant $\chi$ (equals to $2.6805 \times 10^{-3} \mathrm{~mol}^{1 / 3}$ at the pressure $P=0.1 \mathrm{MPa}$ ) does not depend on the nature of the considered liquids.

The choice of a monomolecular layer as a system whose properties determine the surface tension [1] was based on the fact that for near-boiling liquids the surface boundary layers practically do not interact with the molecules from their volume phases. The absence of such an interaction follows from a significant increase in the average distances between the liquid layers, especially at temperatures $T$ close to the boiling points. It should be noted that at $T$, significantly lower than the boiling point $T_{b}$, the surface tension becomes depending on the interaction of the upper surface layer with the nearest one [2].

Since in the present work we study the surface tension of boiling n-alkanes, one can expect that this quantity is also determined by the inter-molecular attraction within monomolecular surface layer. It is also clear that the electronic nature of this formation can differ from that of the above considered near-boiling liquids with molecular $\pi \sigma^{\star}$-hyperconjugation for which the values of $\sigma$ were found to be related to their molecular electronic structure.

Earlier it was shown that $\sigma$ for different liquids (including the boiling ones) can be described by the following equation (see Equation (5) from [1]):

$$
\sigma=\varepsilon_{0}\left(1-T / T_{c}\right) n
$$

where $n$ is the number of surface liquid molecules that must be removed from their equilibrium positions in order to increase the initial liquid surface by one-unit area. Further, $\varepsilon_{0}$ is the absolute value of the average one-particle zero-point binding energy describing any separate molecule of the surface liquid layer at $T=0 \mathrm{~K}$.

It was also shown [1] that the quantity $\varepsilon_{0}$ is proportional to the absolute value of the one-particle energy of intermolecular dispersion attraction $\left|E_{d}\right|$ :

$$
\varepsilon_{0}=\kappa\left|E_{d}\right|=\varphi N\left|E_{\text {orb }}\right| .
$$

In its turn, $\left|E_{d}\right|$ is directly proportional [9] to the value of $\left|E_{o r b}\right|$, where $E_{o r b}$ is the energy of those MOs which contribute essentially to dispersion attraction. Usually, the given MOs are the HOMOs or the nearest to the HOMOs occupied molecular orbitals [9]. The coefficients of proportionality $\kappa$ and $\varphi$ are supposed 
to be constant for different liquids [1], and $N$ is the number of the atoms, whose atomic orbitals enter the above MOs with great coefficients.

Before determining the number $n$ in Equation (2) one should consider some structural features of the surface monolayer of liquid $n$-alkanes. So, for the wide temperature range of liquid $n$-alkanes it has been shown experimentally [10], [11] and theoretically [12] that the hydrocarbon chains of their molecules, forming this monolayer, are oriented normal to its plane. Such an orientation of the hydrocarbon chains of $n$-alkanes results in the following effects. Firstly, the layer has a certain thickness, and secondly, the terminal one-particle methyl groups are on the opposite sides of the monolayer. Thus, the dispersion attraction, which is responsible for the stability of the considered surface monolayer, acts mainly within its volume (but not on its surfaces as it was described for organic molecules in [1]).

Let us consider in more detail the formation of a new unit surface area of a liquid n-alkane. It can be modeled by removing a straight circular cylinder with a unit base area from its surface layer. The choice of just such a cylinder as the volume to be removed is due to the requirement of a minimum of the total energy necessary to overcome the dispersion attraction between the molecules from its side surface and the neighboring molecules outside the cylinder.

The energy of this intermolecular attraction is proportional to the number of molecules situated on the cylinder's side surface, and therefore, to its side surface area. Therefore, a cylinder with the smallest side surface, namely a straight circular cylinder, will correspond to the minimal total attractive energy.

Since the base of the cylinder is a unit area circle, its radius $r$ is equal to one $\pi^{-1 / 2}$. Then the value of its side surface area $S$ equals to $2 \pi r L$ or $2 \pi^{1 / 2} L$ (where $L$ is the thickness of the surface layer). If the area of one n-alkane molecule is $s$, that the number $n$ in Equation (2) can be determined by the expression:

$$
n=S / s=2 \pi^{1 / 2} L s^{-1} \text {. }
$$

Then, using Equations (3) and (4) we can write Equation (2) for surface tension in the form:

$$
\sigma=2 \pi^{1 / 2} \varphi N\left|E_{\text {orb }}\right|\left(1-T_{b} / T_{c}\right) L s^{-1}
$$

Further, it is to be noted that for the molecules of n-alkanes their carbon atoms are spatially shielded by their hydrogen ones. This circumstance means that the dispersion attraction between the molecules within the liquid surface layer arises mainly due to the $\mathrm{H} \cdots \mathrm{H}$ interactions of the neighboring molecules. Therefore, for the studied n-alkanes the number $N$ in Equation (5) is equal to the total number of the hydrogen atoms entering the above considered occupied MO with its orbital energy $E_{o r b}$. One can expect that the dispersion attraction will be the strongest if this MO has the following properties. Firstly, the number of the hydrogen atoms entering it should be as high as possible. Secondly, all the hydrogen atomic orbitals (AOs) belonging to this $\mathrm{MO}$ must have the same sign (+ or -). Thirdly, the given MO has to be either the highest occupied MO 
(HOMO) or one of the occupied MOs which is energetically close to the HOMO [9]. In the considered case of $n$-alkanes, it is this specific molecular orbital (hereinafter we shall denote it as the SMO) that makes the maximum contribution to the dispersive interaction of any pair of neighboring molecules.

\subsection{Relationship "n-Alkanes Electronic Structure-Reaction Rate Constants with $\mathrm{OH}$ Radicals"}

In the second part of the present work we investigate the QSAR between the n-alkanes electronic structure and the rate constants $k$ in their reactions with hydroxyl radicals:

$$
\mathrm{R}_{1}-\left(\mathrm{CH}_{2}\right)-\mathrm{R}_{2}+\mathrm{HO}^{\bullet}=\mathrm{R}_{1}-\left(\mathrm{C}^{\bullet} \mathrm{H}\right)-\mathrm{R}_{2}+\mathrm{H}_{2} \mathrm{O}
$$

Here $R_{1}$ and $R_{2}$ are linear alkyl substituents (or one hydrogen atom together with one methyl substituent in the case of ethane).

In order to find the sought expression connecting the rate constants $k$ of the studied reactions (i) with the particularities of $n$-alkanes' electronic structure we use the Eyring equation from the transition state theory [13]:

$$
k=\left[\tau\left(k_{B} T\right) / h\right] \exp (\Delta S / R) \exp (-\Delta H / R T) .
$$

In Equation (6) $k_{B}$ and $h$ are the Boltzmann and Planck's constants, respectively, $\tau$ is the transmission coefficient of the reactions (i), $\Delta S$ and $\Delta H$ are, respectively, the entropy and the enthalpy of activation of the transition states for the reactions (i), $T$ is the reaction absolute temperature, and $R$ is the universal gas constant. Further, using the explicit form of $\Delta H$, Equation (6) can be written as:

$$
k=\left\{\left[\tau\left(k_{B} T\right) / h\right] \exp (\Delta S / R) \exp (-P \Delta V / R T)\right\} \exp (-\Delta E / R T)
$$

where $\Delta V$ is the change of the volume accompanying the formation of the transition state, $\Delta E$ is the activation energy, and $P$ is the normal atmospheric pressure $(P=0.1 \mathrm{MPa}=$ const $)$.

Moreover, our DFT calculations have shown that the structures of the transition states of all the reactions (i) have some common spatial domain in which hydrogen atom transfer is being realized (see below). Therefore, it is reasonable to assume that the quantities $\tau, \Delta S$, and $\Delta V$ in Equation (7) are constant for all the studied $n$-alkanes. Since the absolute temperature of the reactions (i) is also constant ( $T=297 \pm 2 \mathrm{~K}$ ), so we can present Equation (7) in the following form:

$$
k=A \exp (-\Delta E / R T) \text {, }
$$

where $A$ denotes the expression in its curly braces; therefore, $A$ is constant too. In the logarithmic form Equation (8) may be written as:

$$
\log k=\log A-\Delta E / R T=C+D \Delta E,
$$

where $C=\log A$ and $D=-1 /(R T)$ are some constant coefficients.

Further, as the absolute values of the atomic charges on the hydrogen atoms of 
the n-alkanes are near to zero, so the reactions (i) belong to the type of orbital-controlled reactions. To estimate the quantity $\Delta E$ we have used the expression given by Klopman in his work [14] (see also the work [5]) which describes the energy change of two reactants in the orbital-controlled interactions:

$$
\Delta E=\left[2\left(\beta c_{i} c_{j}\right)^{2}\right] /\left(E_{i}-E_{j}\right) .
$$

In Equation (10) $E_{i}$ is the energy of the molecular orbital of the hydroxyl radical, on which its unpaired electron is located, $E_{j}$ is the energy of the SMO ( $E_{j}=$ $E_{o r b}$, see above) for each investigated alkane, $c_{i}$ and $c_{j}$ are the LCAO coefficients of the atoms entering the $\mathrm{H}$-atom transfer domain, and $\beta$ is the resonance integral between the corresponding atomic orbitals. Therefore, one can assume that the value $2\left(\beta \cdot c_{i} \mathcal{C}_{j}\right)^{2}$ in Equation(10) remains constant for all the $\mathrm{n}$-alkanes under study. In this case, Equation (9) for reaction rate constants has the following final form:

$$
\log k=C+Z /\left(E_{i}-E_{\text {orb }}\right),
$$

where the constant $Z=D \cdot\left[2\left(\beta \cdot c_{i} c_{j}\right)^{2}\right]$.

\section{Computational Methods}

The electronic structures of all the considered $n$-alkanes in their optimized conformations were calculated by the DFT (B3LYP) method [15] with the 6 - 31 $\mathrm{G}(\mathrm{d})$ basis set using the package of quantum chemical programs GAUSSIAN09 [16].

\section{Results and Discussion}

\subsection{Surface Tension of the n-Alkanes}

In the present work we have studied the series of the $\mathrm{n}$-alkanes presented in Table 1. All these compounds contain linear hydrocarbon chains. The geometry structure calculations show that their optimal conformations have planar hydrocarbon chains for which any four consecutive atoms are in a trans-configuration. Analysis of the occupied MOs of the studied compounds showed that for all the n-alkanes examined, their HOMOs do not satisfy the above criteria for revealing the MOs with the energy $E_{\text {orb }}$. It follows from the fact that the HOMOs are spatially shielded by the hydrogen atoms of the methylene groups, and, therefore, these orbitals cannot contribute to the value of the surface tension (Figure 1). The exceptions are ethane and propane where the HOMOs include the methylene hydrogen atoms discussed above, but their AOs enter the HOMOs with different signs (Figure 1).

At the same time, for all the studied n-alkanes, we found the such occupied specific MOs denoted earlier as the SMOs which satisfies the above criteria. They include (with the same sign) the 1s-AOs of the hydrogen atoms of all methylene groups, and their energies $E_{\text {orb }}$ are close to the energies of the corresponding HOMOs. The corresponding HOMOs and the SMOs for n-propane, n-pentane, 


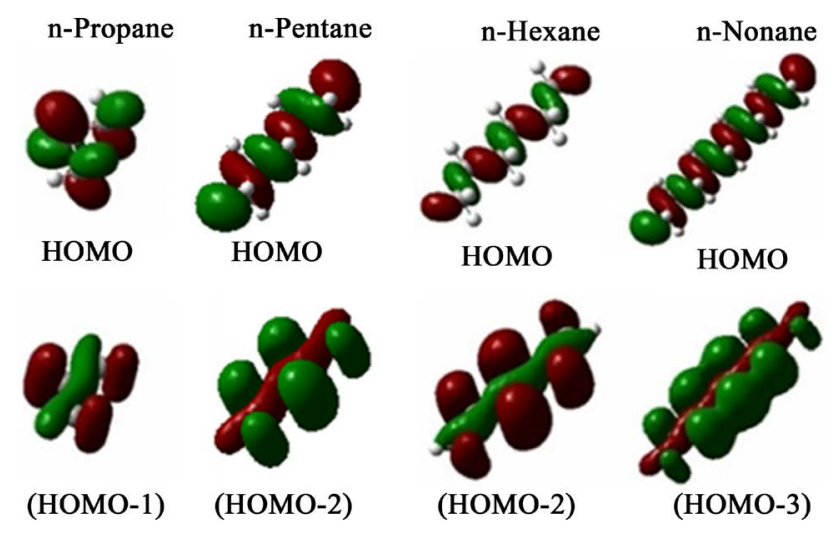

Figure 1. The view of the HOMOs (the upper row) and the SMOs (the lower row) for some of the studied n-alkanes.

n-hexane, and n-nonane are shown in Figure 1.

Further, for any investigated n-alkane molecule we consider that the number of its methylene groups (denoted as $N_{m}$ ) is equal to the number of its carbon atoms. It means that its two terminal methyl groups are considered hereafter as the corresponding methylene ones connected with the two end hydrogen atoms belonging to its hydrocarbon chain. Then, the number of the hydrogen atoms $\mathrm{N}$ entering the SMO of any n-alkane will be equal to $2 N_{m}$. Besides, since the length of one hydrocarbon chain is proportional to $N_{m}$, then the layer thickness $L$ in Equation (5) will be also proportional to $N_{m}$ due to the normal orientation of the molecules to the two planar sides of the surface monolayer [10] [11] [12]:

$$
L=\delta N_{m} \text {. }
$$

Further, we assume that the molecules of the surface layer do not leave it for some time, which is large enough to neglect their movement in the normal direction to the two boundary planes of the monolayer. However, these molecules can possess some libration motion changing the angle between their hydrocarbon chains and this normal. The amplitude $A$ of these librations will be also proportional to $N_{m}$ (if we pass from one n-alkane to another one):

$$
A=\lambda N_{m} \text {. }
$$

Using the principle of corresponding states and bearing in mind that we deal with the boiling liquids (whose reduced boiling temperatures are very close) we suppose that each of the proportionality coefficients $\delta$ and $\lambda$ in Equations (12), (13) is the same for all the studied n-alkanes. We can also assume that the average one-particle area $s$ in Equation (5) is approximately equal to the area of some rectangle with the sides $L$ and $2 A$ :

$$
s=2 A L=\omega\left(N_{m}\right)^{2},
$$

where the proportionality coefficient $\omega=2 \delta \lambda$ is constant for any of the studied n-alkanes.

Substituting the defined quantities $L, S$, and $N=2 N_{m}$ into Equation (5), we obtain the following expression for the surface tension: 


$$
\begin{aligned}
\sigma & =\left[4 \pi^{1 / 2} \varphi N_{m}\left|E_{\text {orb }}\right|\left(1-T_{b} / T_{c}\right) \delta N_{m}\right] \times\left[\omega\left(N_{m}\right)^{2}\right]^{-1} \\
& =\xi\left|E_{\text {orb }}\right|\left(1-T_{b} / T_{c}\right)=\xi G
\end{aligned}
$$

Here the coefficient $\xi$ includes the multiplier $4 \pi^{1 / 2}$ and the constants $\varphi, \delta$, and $\tau$, and the quantity $G=\left|E_{\text {orb }}\right|\left(1-T_{b} / T_{c}\right)$. Here it is to be noted that the number of methyl groups $N_{m}$ is not presented in the final Equation (15). Now we have to take into account the influence on $\sigma$ of the two end hydrogen atoms which belong to the hydrocarbon chain and do not enter the number $2 N_{m}$ (see above). These hydrogen atoms are on the different sides of the surface monolayer, and they enter the corresponding SMOs for all the molecules forming the surface monolayer (Figure 1). It means that the interaction between these hydrogen atoms of the neighboring molecules can make some additional contribution to the surface tension. Denoting this contribution as $\rho$ and considering that $\rho$ is a constant for all the investigated n-alkanes, we can rewrite Equation (15) in the form:

$$
\sigma=\xi G+\rho .
$$

To define the constants $\xi$ and $\rho$ we have studied the set of the boiling short chain n-alkanes presented in Table 1 . The experimental values of $T_{b}, T_{c}$ and $\sigma$ were taken from [17] [18] [19] [20] [21]. Table 1 also contains the calculated values of $\left|E_{\text {orb }}\right|$ which are required for the calculation of $G=\left|E_{\text {orb }}\right|\left(1-T_{b} / T_{c}\right)$. The constants $\xi$ and $\rho$ can be obtained from the linear regression equation obtained by the least square method:

$$
\sigma \times 10^{3}=0.0448 G+0.8333 .
$$

The values of $\sigma$ calculated by means of Equation (17) are also given in Table 1 along with the values of $\sigma$ obtained by the so-called "Leave-one-out" Cross-Validation

\begin{tabular}{|c|c|c|c|c|c|c|c|c|}
\hline \multirow{2}{*}{$N$} & \multirow{2}{*}{ Alkane } & \multirow{2}{*}{$T\left({ }^{\bullet} \mathrm{K}\right)$} & \multirow{2}{*}{$T_{c}\left({ }^{\bullet} \mathrm{K}\right)$} & \multirow{2}{*}{$\begin{array}{c}\left|E_{\text {orb }}\right| \\
(\mathrm{kJ} / \mathrm{mol})\end{array}$} & \multirow{2}{*}{$\begin{array}{c}G \\
(\mathrm{~kJ} / \mathrm{mol})\end{array}$} & \multicolumn{3}{|c|}{$\sigma \times 10^{3}(\mathrm{~N} / \mathrm{m})$} \\
\hline & & & & & & Exp. & Calc. & LOOCV \\
\hline 1 & Ethane & 184.55 & 305.32 & 893.58 & 353.4580 & 16.31 & 16.67 & 16.99 \\
\hline 2 & Propane & 231.11 & 369.83 & 873.56 & 327.6656 & 15.50 & 15.51 & 15.53 \\
\hline 3 & Butane & 272.65 & 425.12 & 863.34 & 309.6393 & 14.93 & 14.71 & 14.67 \\
\hline 4 & Pentane & 309.22 & 469.70 & 845.16 & 288.7624 & 14.07 & 13.77 & 13.72 \\
\hline 5 & Hexane & 341.88 & 507.60 & 841.27 & 274.6572 & 13.41 & 13.14 & 13.12 \\
\hline 6 & Heptane & 371.58 & 540.20 & 837.02 & 261.2699 & 12.45 & 12.54 & 12.57 \\
\hline 7 & Octane & 398.83 & 568.70 & 835.47 & 249.5532 & 12.09 & 12.01 & 11.99 \\
\hline 8 & Nonane & 423.97 & 594.60 & 833.73 & 239.2533 & 11.53 & 11.55 & 11.56 \\
\hline 9 & Decane & 447.30 & 617.70 & 833.00 & 229.7927 & 10.75 & 11.13 & 11.29 \\
\hline
\end{tabular}
Scheme (LOOCV). The given statistical method consists in consequent removal

Table 1. The data necessary to estimate the constants $\xi$ and $\rho$ in Equation (16) and the subsequent calculation of $\sigma$.

*The experimental values of $T_{b}, T_{c}$ and $\sigma$ were taken from [17] [18] [19] [20] [21]. 


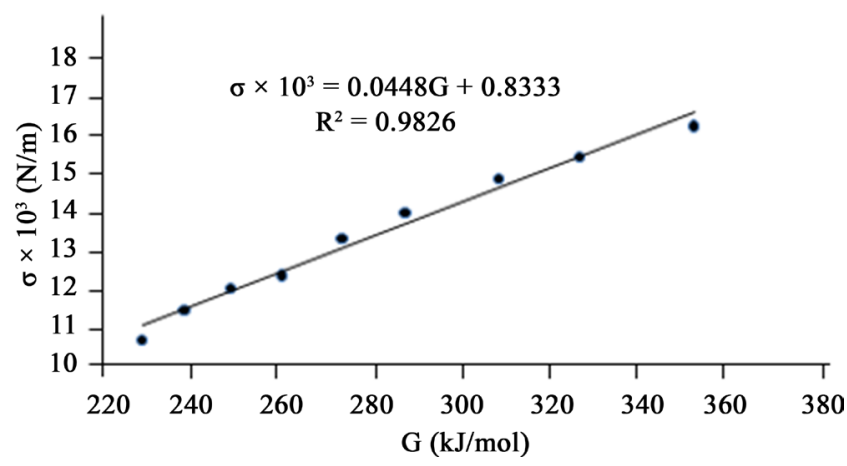

Figure 2. The correlation between the experimental values of $\sigma$ and the calculated values of $G$.

of one of the studied compounds from the regression analysis and the following calculation of the predicted quantity for the removed compound. It can be seen that the values of the surface tension in the last two columns of Table 1 are very close to each other and to the corresponding experimental ones. Note also that the constant $\rho=0.8333$ in Equation (17) is relatively small in comparison with the amount of $0.0448 G$. This fact reflects the small effect of the end hydrogen atoms on $\sigma$.

The plot of the correlation between the experimental values of $\sigma \times 10^{3}\left[\mathrm{~N} \cdot \mathrm{m}^{-1}\right]$ and the calculated parameter $G\left[\mathrm{~kJ} \cdot \mathrm{mol}^{-1}\right]$ is presented in Figure 2.

\subsection{Rate Constants for the Reactions$$
\mathbf{R}_{1}-\left(\mathrm{CH}_{2}\right)-\mathrm{R}_{2}+\mathrm{HO}^{\circ}=\mathbf{R}_{1}-\left(\mathrm{C}^{\circ} \mathrm{H}\right)-\mathrm{R}_{2}+\mathrm{H}_{2} \mathrm{O}
$$

First of all, it is to be noted, that our DFT calculations have shown, that, for all the studied n-alkanes, it is these SMOs which determine the values of their surface tension (Section 4.1), are also decisive in their interaction with hydroxyl radicals. So, Figure 3 demonstrates the interaction of the SMO of n-pentane (Figure 3(a)) with the hydroxyl radical $\mathrm{HO}^{\bullet}$. This interaction generates a transition state characterized by the formation of a three-center $\mathrm{C}-\mathrm{H}-\mathrm{O}$ bond (see Figure 3(b)), which can be considered as some spatial domain where the transfer of a hydrogen atom takes place. Redistribution of the electronic density within this $\mathrm{C}-\mathrm{H}-\mathrm{O}$ bond leads to the elongation of the $\mathrm{C}-\mathrm{H}$ bond and to the simultaneous formation of the new $\mathrm{O}-\mathrm{H}$ one. Both these processes result in the cleavage of the $\mathrm{C}-\mathrm{H}$ bond and the appearance of one water molecule and a n-penthyl radical (Figure 3(c)).

In Section 4.2 it was found the formulae $\log k=C+Z /\left(E_{i}-E_{\text {orb }}\right)$, which connects the rate constants $k$ of the above reactions $(i)$ and the energies of the corresponding SMOs. In order to find parameters $C, Z$, and $E_{i}$ which are common for all the n-alkanes, we have used the experimental values of $k$ taken from the works [22]-[30] and presented in Table 2. The following multiple regression analysis allows one to determine the above parameters. The corresponding numerical values of $C, Z$, and $E_{i}$ are equal to $8.1254,496.25 \mathrm{~kJ} / \mathrm{mol}$, and 1003.13 $\mathrm{kJ} / \mathrm{mol}$, respectively. The values of $\log k$ calculated by means of Equation (11) 


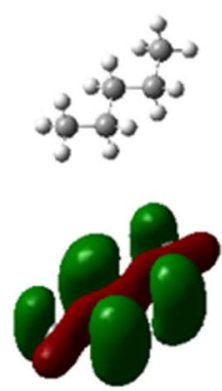

(a) SMO (HOMO-2)

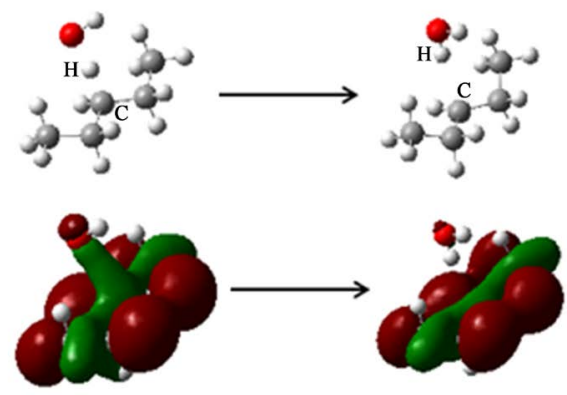

(b) $\mathrm{R}(\mathrm{C}-\mathrm{H})=12.6 \AA$

Figure 3. Formation of three-center $\mathrm{C}-\mathrm{H}-\mathrm{O}$ bonding $\mathrm{MO}$ between the SMO of n-pentane and the unpaired electron of hydroxyl radical.

Table 2. The data necessary to estimate the constants $C, Z$, and $E_{i}$ inEquation (11) and the subsequent calculation of $-\log k$.

\begin{tabular}{cccccc}
\hline $\boldsymbol{N}$ & Alkane & $\begin{array}{c}\boldsymbol{k}_{\text {exp. }} \times 1 \mathbf{1 0}^{12} \\
\left(\mathrm{~cm}^{3} \cdot \mathrm{mol}^{-1} \cdot \mathbf{s}^{-1}\right)\end{array}$ & $-\log k_{\text {exp. }}$ & $\begin{array}{c}\left|E_{\text {orb }}\right| \\
\left(\mathbf{k J} \cdot \mathrm{mol}^{-1}\right)\end{array}$ & $-\log k_{\text {calc. }}$ \\
\hline 1 & Ethane & 0.222 & 12.65 & 893.58 & 12.66 \\
2 & Propane & 1.12 & 11.95 & 873.56 & 11.95 \\
3 & Butane & 2.10 & 11.68 & 863.34 & 11.68 \\
4 & Pentane & 5.30 & 11.28 & 845.16 & 11.27 \\
5 & Hexane & 5.91 & 11.22 & 841.27 & 11.19 \\
6 & Heptane & 7.26 & 11.14 & 837.02 & 11.11 \\
7 & Octane & 8.24 & 11.08 & 835.47 & 11.09 \\
8 & Nonane & 9.36 & 11.03 & 833.73 & 11.05 \\
9 & Decane & 9.90 & 11.00 & 833.00 & 11.04 \\
\hline
\end{tabular}

with the above parameters are presented in the last column of Table 2. As it is seen, the calculated values of $\log k_{\text {calc }}$ practically coincide with the corresponding experimental ones. Moreover, these values $\log k_{\text {calc }}$ are in a rather good agreement with the latest experimental data [31] [32]. Thus, one can conclude, that Equation (11) describes adequately the relationship between the reaction rate constants $k$ and the energies of the above n-alkanes specific MOs.

\section{Conclusions}

For the series of short chain n-alkanes we have identified the occupied Specific Molecular Orbitals (SMOs) which play a decisive role in determining their various physical and chemical properties: the surface tension $\sigma$ near their normal boiling points and the reaction rate constants $k$ with hydroxyl radicals (at $297 \pm$ $2 \mathrm{~K}$ ). Basing on the theoretical consideration of the intermolecular dispersion attraction in the surface layers and the orbital controlled interactions for the reactions $\mathrm{R}_{1}-\left(\mathrm{CH}_{2}\right)-\mathrm{R}_{2}+\mathrm{HO}^{\circ}=\mathrm{R}_{1}-\left(\mathrm{C}^{*} \mathrm{H}\right)-\mathrm{R}_{2}+\mathrm{H}_{2} \mathrm{O}$, the two quantitative relationship were received. They connect the energy values $\left|E_{\text {orb }}\right|$ of these SMOs with the above quantities $\sigma$ and $k$. The calculated values of $\sigma$ and $k$ are in fairly 
good agreement with the corresponding experimental data.

Obviously, the results of the present work can be also applied, for example, to cyclo-alkanes and other organic compounds, reacting with free radicals other than $\mathrm{OH}^{\circ}$. It can also be assumed that the revealed role of the SMOs in the aforementioned dispersion attraction may serve for understanding of the nature of evaporation and condensation of the hydrocarbons under consideration. These problems will be considered in our next works.

\section{Conflicts of Interest}

The authors declare no conflicts of interest regarding the publication of this paper.

\section{References}

[1] Gorbachev, M.Yu., Gorinchoy, N.N. and Arsene, I. (2017) Surface Tension of Near-Boiling Liquids with Intramolecular $\pi \sigma^{\star}$-Hyperconjugation: DFT Calculations. Journal of Molecular Liquids, 237, 108-112. https://doi.org/10.1016/j.molliq.2017.04.069

[2] Gorbachev, M.Yu., Budei, O., Arsene, I. and Gorinchoy, N.N. (2014) Surface Tension of Liquids with $\pi$-Conjugated Planar Molecules: DFT Calculation. Journal of Molecular Liquids, 190, 50-53. https://doi.org/10.1016/j.molliq.2013.10.017

[3] Panić, J., Tot, A., Janković, N., Drid, P., Gadžurić, S. and Vraneš, M. (2020) Physicochemical and Structural Properties of Lidocaine-Based Ionic Liquids with Anti-Inflammatory Anions. RSC Advances, 10, 14089-14098. https://doi.org/10.1039/C9RA08815F

[4] Md. Abdur Rauf, S., Arvidsson, P.I., Albericio, F., Govender, T., Maguire, G.E.M., Kruger, H.G. and Honarparvar, B. (2015) The Effect of n-Methylation of Amino Acids (Ac-X-OMe) on Solubility and Conformation: a DFT Study. Organic \& Biomolecular Chemistry, 13, 9993-10006. https://doi.org/10.1039/C5OB01565K

[5] Nolte, T.N. and Peijnenburg, W.J.G.M. (2018) Use of Quantum-Chemical Descriptors to Analyse Reaction Rate Constants between Organic Chemicals and Superoxide/Hydroperoxyl (O2*-/HO2*). Free Radical Research, 52, 1118-1131. https://doi.org/10.1080/10715762.2018.1529867

[6] Marc, G., Stana, A., Oniga, S.D., Pîrnău, A., Vlase, L. and Oniga, O. (2019) New Phenolic Derivatives of Thiazolidine-2,4-dione with Antioxidant and Antiradical Properties: Synthesis, Characterization, in Vitro Evaluation, and Quantum Studies. Molecules, 24, 2060. https://doi.org/10.3390/molecules24112060

[7] Rios, C. and Salcedo, R. (2012) Computational Termochemistry Study of the C80 Isomers and Their Endo Lanthanum Complexes by Applying Homodesmotic and Isodesmic Reactions. Molecules, 17, 14588-14601.

https://doi.org/10.3390/molecules 171214588

[8] Da Silva, R.R., Ramalho, T.C., Santos, J.M. and Figueroa-Villar, J.D. (2006) On the Limits of Highest-Occupied Molecular Orbital Driven Reactions: The Frontier Effective-for-Reaction Molecular Orbital Concept. The Journal of Physical Chemistry $A, 110,1031-1040$. https://doi.org/10.1021/jp054434y

[9] Gavezzotti, A. (2007) Supramolecular Interactions: Energetic Consideration. In: Braga, D. and Grepioni, F., Eds., Making Crystals by Design: Methods, Techniques and Applications, Wiley-VCH Verlag GmbH Co. KGaA, Weinheim, 1-24. 
[10] Esenturk, O. and Walker, R.A. (2006) Surface Vibrational Structure at Alkane Liquid/Vapor Interface. The Journal of Chemical Physics, 125, Article ID: 174701. https://doi.org/10.1063/1.2356858

[11] Wu, X.Z., Sirota, E.B., Sinha, S.K. and Ocko, B.M. (1993) Surface Crystallization of Liquid Normal-Alkanes. Physical Review Letters, 70, 958-961. https://doi.org/10.1103/PhysRevLett.70.958

[12] Kawamata, M. and Yamamoto, T. (1997) Molecular Dynamics Simulation of Surface Ordering in Liquid n-Alkanes. Journal of the Physical Society of Japan, 66, 2350-2354. https://doi.org/10.1143/JPSJ.66.2350

[13] Eyring, H. (1935) The Activated Complex in Chemical Reactions. The Journal of Chemical Physics, 3, 107-115. https://doi.org/10.1063/1.1749604

[14] Klopman, G. (1968) Chemical Reactivity and the Concept of Charge- and Frontier-Controlled Reactions. Journal of the American Chemical Society, 90, 223-234. https://doi.org/10.1021/ja01004a002

[15] Lee, C., Yang, W. and Parr, R.G. (1988) Development of the Colle-Salvetti Correlation-Energy Formula into a Functional of the Electron Density. Physical Review $B$, 37, 785-789. https://doi.org/10.1103/PhysRevB.37.785

[16] Frisch, M.J., Trucks, G.W., Schlegel, H.B., Scuseria, G.E., Robb, M.A., Cheeseman, J.R., et al. (2009) Gaussian 09, Revision B.01. Gaussian, Inc., Wallingford.

[17] Dean, J.A. (1987) Handbook of Organic Chemistry. McGraw-Hill Book Company, New York.

[18] Yaws, C.L. (2008) Thermophysical Properties of Chemicals and Hydrocarbons. William Andrew, Norwich.

[19] Frőba, A.P., Penedo-Pellegrino, L. and Leipertz, A. (2004) Viscosity and Surface Tension of Saturated n-Pentane. International Journal of Thermophyics, 25, 1323-1337. https://doi.org/10.1007/s10765-004-5741-1

[20] Beaton, C.F. and Hewitt, G.F. (1989) Physical Property Data for the Design Engineer. Hemisphere Publishing Corporation, New York.

[21] Nikolsky, B.P. (1963) Chemist's Handbook, Vol. 1, Chemistry, Moscow.

[22] Atkinson, R. (2003) Kinetics of the Gas-Phase Reactions of OH Radicals with Alkanes and Cycloalkanes. Atmospheric Chemistry and Physics, 3, 2233-2307. https://doi.org/10.5194/acp-3-2233-2003

[23] Atkinson, R., Aschmann, S.M., Winer, A.M. and Pitts Jr., J.N. (1982) Rate Constants for the Reaction of $\mathrm{OH}$ Radicals with a Series of Alkanes and Alkenes at $299 \pm 2 \mathrm{~K}$. International Journal of Chemical Kinetics, 14, 507-516. https://doi.org/10.1002/kin.550140508

[24] Atkinson, R., Aschmann, S.M., Carter, W.P.L., Winer, A.M. and Pitts Jr., J.N. (1982) Kinetics of the Reactions of $\mathrm{OH}$ Radicals with n-Alkanes at $299 \pm 2 \mathrm{~K}$. International Journal of Chemical Kinetics, 14, 781-788. https://doi.org/10.1002/kin.550140706

[25] Abbatt, J.P.D., Demerjian, K.L. and Anderson, J.G. (1990) A New Approach to Free-Radical Kinetics: Radially and Axially Resolved High-Pressure Discharge Flow with Results for Hydroxyl + (Ethane, Propane, n-Butane, n-Pentane). Fwdarw. Products at 297 K. The Journal of Physical Chemistry, 94, 4566-4575. https://doi.org/10.1021/j100374a039

[26] Behnke, W., Hollander, W., Koch, W., Nolting, F. and Zerzsch, C. (1988) A Smog Chamber for Studies of the Photochemical Degradation of Chemicals in the Presence of Aerosols. Atmospheric Environment, 6, 1113-1120. https://doi.org/10.1016/0004-6981(88)90341-1 
[27] Chuong, B. and Stevens, P.S. (2002) Measurements of the Kinetics of the OH-Initiated Oxidation of Isoprene. Journal of Geophysical Research, 107, ACH 12-1-ACH 12-10. https://doi.org/10.1029/2001JD000865

[28] Cox, R.A., Derwent, R.G. and Williams, M.R. (1980) Atmospheric Photooxidation Reactions. Rates, Reactivity, and Mechanism for Reaction of Organic Compounds with Hydroxyl Radicals. Environmental Science \& Technology, 14, 57-61. https://doi.org/10.1021/es60161a007

[29] Barnes, I., Bastian, V., Becker, K.H., Fink, E.H. and Nelsen, W. (1986) Oxidation of Sulphur Compounds in the Atmosphere: I. Rate Constants of OH Radical Reactions with Sulphur Dioxide, Hydrogen Sulphide, Aliphatic Thiols and Thiophenol. Journal of Atmospheric Chemistry, 4, 445-466. https://doi.org/10.1007/BF00053845

[30] Ferrari, C., Roche, A., Jacob, V., Foster, P. and Baussand, P. (1996) Kinetics of the Reaction of $\mathrm{OH}$ Radicals with a Series of Esters, under Simulated Conditions at 295 K. International Journal of Chemical Kinetics, 28, 609-614. https://doi.org/10.1002/(SICI)1097-4601(1996)28:8<609::AID-KIN6>3.0.CO;2-Z

[31] Han, L., Siekmann, F. and Zetzsch, C. (2018) Rate Constants for the Reaction of OH Radicals with Hydrocarbons in a Smog Chamber at Low Atmospheric Temperatures. Atmosphere, 9, 1-16. https://doi.org/10.3390/atmos9080320

[32] Crawford, M.A., Dang, B., Hoang, J. and Li, Zh. (2011) Kinetic Study of OH Radical Reaction with n-Heptane and n-Hexane at 240-340K Using the Relative Rate/Discharge Flow/Mass Spectrometry (RR/DF/MS) Technique. International Journal of Chemical Kinetics, 43, 489-497. https://doi.org/10.1002/kin.20574 